DOI: $10.5216 /$ racs.v5.65682

\title{
Docencia, interculturalidad y diversidad cultural en el aula: una propuesta pedagógica
}

\author{
Maria Luisa Matus Pineda ${ }^{1}$
}

\section{RESUMEN}

En el presente documento me centraré en describir los fundamentos metodológicos de una propuesta pedagógica para la atención de la diversidad cultural en el aula, fruto de una investigación que se llevó acabo en una escuela primaria bilingüe de la comunidad ikoot (huave) de San Mateo del Mar, Oaxaca, México. Dicha propuesta sugiere que las actividades comunitarias y la visibilización de los aprendizajes sociculturales infantiles relacionados con la actividad pesquera, se conviertan en el punto de partida para la transverzalización de los conocimientos escolares, es decir, que se desarrollen procesos pedagógicos socioculturalmente situados y que den pauta hacia una educación pertinente.

PALABRAS CLAVE: Diversidad cultural. Práctica docente. Metodologías interculturales.

\section{Teaching, interculturality and cultural diversity in the classroom: a pedagogical proposal}

\begin{abstract}
In this document I will focus on describing the methodological foundations of a pedagogical proposal for the care of cultural diversity in the classroom, the result of an investigation that was carried out in a bilingual primary school of the ikoot (huave) community of San Mateo del Mar, Oaxaca, Mexico. This proposal suggests that community activities and the visibility of children's socicultural learning related to fishing activity, become the starting point for the transverzalization of school knowledge, that is, that socioculturally situated pedagogical processes are developed and that give way to a pertinent education.
\end{abstract}

KEYWORDS: Cultural diversity. Teaching practice. Intercultural methodologies.

\footnotetext{
${ }^{1}$ Centro De Investigaciones y Estudios Superiores en Antropologia Social (CIESAS), Oaxaca, México. E-mail: luisa_m21@hotmail.com.

Dossiê Redes de Inter-aprendizaje: nuevas cartografías interculturales y algunas propuestas de transformación

R. Articul.const.saber, 2020, v.5: e65682
} 


\section{Introducción}

Me centraré en describir una propuesta pedagógica para la atención de la diversidad cultural en el aula, fruto de una investigación que se llevó acabo en una escuela primaria bilingüe de la comunidad ikoot (huave) de San Mateo del Mar², Oaxaca, México. La Escuela Primaria Bilingüe Adolfo López Mateos, es una de las instituciones educativas de organización completa que atiende a los niños y las niñas en edad escolar en la comunidad de San Mateo del Mar, Oaxaca. En el momento de la investigación, excepto un docente, todos eran oriundos de la comunidad, hablantes del ombeayiiüts ${ }^{3}$ y contaba con una formación enfocada a la atención de la educación para el medio indígena en dos instituciones formadoras de docentes ${ }^{4}$. Se pudo observar que dicha lengua se usa como lengua de instrucción en el primer ciclo $\left(1^{\circ}\right.$ y $2^{\circ}$ grado $)$, minimizando su uso en el segundo y tercer ciclo $\left(3^{\circ}-6^{\circ}\right)$ y está presente como lengua de comunicación en la escuela y fuera de ella.

Los docentes de la Escuela Primaria Bilingüe en mención, participaban activamente en el PTEO 5 , que conjuntamente con el Instituto Estatal de Educación Pública de Oaxaca

\footnotetext{
${ }^{2}$ San Mateo del Mar, es una comunidad lacustre con alta vitalidad lingüística que se ubica en el Istmo Oaxaqueño, al sureste de la república mexicana. La pesca es su principal actividad económica, de donde obtienen principalmente productos como el pescado, el camarón y la jaiba, que son consumidos localmente y comercializados en puntos estratégicos de la región.

${ }^{3}$ Es la forma en la que autonombran la lengua huave.

${ }^{4}$ La mayoría de los docentes eran egresado(a)s de la Licenciatura en Educación Primaria para el Medio Indígena de la Universidad Pedagógica Nacional (UPN) 201, uno más de la Licenciatura en Educación Primaria Intercultural Bilingüe de la Escuela de la Escuela en la Escuela Normal Bilingüe e Intercultural del Estado de Oaxaca (ENBIO) y por último un egresado del bachillerato que en se encontraba estudiando la Licenciatura en Educación Primaria para el Medio Indígena de la UPN en mención.

${ }^{5}$ El PTEO surgió en el 2013 como una propuesta piloto masiva en el estado de Oaxaca en los niveles educativos preescolar y primaria. En su discurso, esta propuesta enfatiza la articulación de la escuela con la comunidad, donde se contempla la incorporación de los saberes comunitarios en las prácticas pedagógicas, a fin de transformar las relaciones verticales características del sistema educativo en el estado de Oaxaca (PTEO, 2013). Este plan lo creó la sección XXII del Sindicato Nacional de Trabajadores de la Educación (SNTE) -considerado como uno de los sindicatos con amplia trayectoria en Oaxaca- y el gobierno estatal como una alternativa a la Alianza por la Calidad de la Educación (ACE) del Gobierno Federal (IEEPO-SNTE, 2013). La ACE es una alianza firmada entre el Gobierno Federal y los maestros de México representados por el Sindicato de Trabajadores de la Educación (SNTE) con el objetivo de impulsar la transformación en la calidad educativa. Sus acciones se plantearon en 5 ejes: 1. Modernización de los centros escolares, 2. Profesionalización de los maestros y autoridades educativas, 3. Bienestar y desarrollo integral de los alumnos. Más que una alianza educativa fue

Dossiê Redes de Inter-aprendizaje: nuevas cartografías interculturales y algunas propuestas de transformación R. Articul.const.saber, 2020, v.5: e65682
} 
(IEEPO), impulsan en las escuelas la realización de proyectos escolares que incluyan a otros tipos de actores: la comunidad y los padres de familia. Dentro de estos proyectos, centré mi atención en la actividad pesquera que constituye la actividad comunitaria predominante y con la que se busca un engranaje entre la cultura escolar y la cultura comunitaria.

A pesar de las condiciones culturales y de formación de los docentes, ellos exigían un acompañamiento que los apoyara a construir un punto de encuentro entre la normatividad, la institucionalidad y la resolución de problemas prácticos cotidianos en un contexto con alta vitalidad lingüística, cultural y etno-política. Insisten en la importancia de sistematizar sus estrategias pedagógicas aunque, percatándose de sus límites, aceptan que no saben del todo cómo hacerlo.

En este sentido, se propone una herramienta didáctica con base en el Método Inductivo Intercultural (MII), en donde el centro del acto pedagógico lo constituye la actividad pesquera y la visibilización de los aprendizajes sociculturales infantiles relacionadas con dicha actividad. Lo anterior podría ser el punto de partida para la transverzalización de los conocimientos escolares con los conocimientos comunitarios, con el fin de contribuir en el desarrollo de procesos pedagógicos socioculturalmente situados que den pauta hacia una educación pertinente. Lo anterior se logró gracias a los principales elementos que sustentan el Método Inductivo Intercultural (MII) propuesto por GASCHÉ (2008), como una herramienta pedagógica para explicitar por medio de la colaboración y el interaprendizaje, los conocimientos implícitos en las actividades pesqueras realizadas por los niños y niñas, a fin de identificar su articulación en un currículum escolar interculturalizado.

\section{La interculturalidad y la diversidad cultural}

considerado un pacto político, de ahí el rechazo hacia dicha propuesta en el estado Oaxaqueño y en otros estados de la república mexicana.

Dossiê Redes de Inter-aprendizaje: nuevas cartografías interculturales y algunas propuestas de transformación R. Articul.const.saber, 2020, v.5: e65682 
Según DIETZ (2010), el rendimiento y el éxito escolar de los niños provenientes de los grupos minoritarios (étnicas, culturales, religiosos, etc), ha sido el punto central para que diferentes países adopten medidas en el campo educativo, en este sentido, “en la percepción de muchos educadores, pedagogos y políticos encargados de las instituciones escolares, el fracaso escolar de estos alumnos reflejan un 'impedimento' o 'déficit' distintivo (GUNDARA, 1982) que a menudo se tiende a equiparar con 'la pertinencia étnica o la condición de inmigrante' (FRANZÉ, 1998:47)" (2010: 69). El enfoque reduccionista de esta visión ha incentivado reformas educativas que se perfilan hacia la integración escolar y la regularización académica, negando la diversidad cultural que se refleja en las aulas.

A partir de la década de los 80, en América Latina 17 países tienen experiencias en Educación Intercultural Bilingüe (EIB), donde se evidencia una agenda de temas pendientes por resolver en este campo que van desde el uso didáctico la lengua indígena y su fortalecimiento (HECHT Y LONON, 2011), hasta el cuestionar los alcances y limitaciones de este tipo de proyecto educativo, que constituye no solamente una propuesta pedagógica para la atención de la diversidad, sino también, una propuesta política. De esta manera, la integración escolar sigue siendo el "desafío" de las adaptaciones compensatorias en los sistemas educativos de diferentes países (DIETZ, 2010, 2019; SCHMEKLES, 2006).

\begin{abstract}
gracias al multiculturalismo la 'intervención pedagógica' indirectamente recupera y reactualiza su histórica misión de estigmatizar a 'lo ageno' para integrar y nacionalizar 'lo propio'. La educación 'multicultural' o 'íntercultural' recurre a este legado pedagógico en su implícita pero frecuente distinción entre 'lo civilizable y lo intrínsecamente malo’ dentro de la relación intercultural (DIETZ, 2010: 70).
\end{abstract}

Sartorello (2009) identifica dos polos de interpretación de la interculturalidad en lo general y de la Educación Intercultural Bilingüe en el medio indígena en particular: por un lado se encuentra la concepción de las políticas interculturales como mecanismos asistencialistas y por el otro, considera que la interculturalidad y la educación intercultural bilingüe son un derecho y medio para lograr la reproducción de las culturas en minoría y en condición subalterna.

La interculturalidad pensada e implementada desde la hegemonía neoliberal se volvería de esa forma un concepto estratégico que sustituye el discurso sobre la pobreza por el discurso sobre la cultura, tratando de esconder el peso y la

Dossiê Redes de Inter-aprendizaje: nuevas cartografías interculturales y algunas propuestas de transformación R. Articul.const.saber, 2020, v.5: e65682 
importancia que tienen la injusticia distributiva, las desigualdades económicas y las relaciones de poder en la construcción de nuevas relaciones interculturales entre Estado y pueblos indígenas en América Latina. El proceso de oficialización y retorización de la interculturalidad, aunado a la concesión de pequeñas cuotas de poder y nuevos - pero limitados - derechos a los pueblos indígenas, serviría entonces para invisibilizar las crecientes asimetrías socio-económicas producto de las políticas neoliberales, resultando por lo tanto funcionales al modelo económico y político hegemónico (SARTORELLO, 2009: 82).

De esta manera, las políticas de estado que respaldan la educación intercultural bilingüe continúan invisibilizando la diversidad cultural presentes en las comunidades indígenas, ya que carecen de una fundamentación empírica con base en estudios etnográficos que demuestre el impacto de lo propuesto. En las prácticas pedagógicas se carece de un verdadero reconocimiento de la alteridad, por lo que "la asimilación, la integración y el pluralismo cultural siguen siendo los grandes modelos educativos dirigidos a la población indígena" (JIMÉNEZ, 2009: 33).

GASCHÉ (2008) considera que en su sentido más amplio, la pretensión de la interculturalidad es el respeto a la alteridad manifestada en los pueblos y comunidades indígenas dentro un territorio y propone que el interaprendizaje (BERTELY Y GASCHÉ, 2011) es la palabra clave que evoca el proceso fundador de todo el proyecto educativo intercultural. En este sentido se propone que exista un nuevo tipo de relación social entre los actores escolares y la comunidad, donde se propicie el empoderamiento a través de prácticas pedagógicas para la transformación de la dominación económica y política de muchos pueblos indígenas.

En este sentido, en el caso de México, existen diversas experiencias educativas bajo control de organizaciones indígenas que han trabajado "desde abajo", con la activa participación de didactas nativos ${ }^{6}$. Estas reflejan el trabajo realizado por una nueva generación de portadores y articuladores de saberes tanto académicos como comunitarios, tanto indígenas

\footnotetext{
${ }^{6}$ Tal es el caso de las Tarjetas de Autoaprendizaje (BERTELY, 2004), de los integrantes tsotsiles, tseltales y chóoles de la Unión de Maestros de la Nueva Educación para México (UNEM) y educadores independientes, y por los jóvenes tseltales del Programa de Educación Comunitaria Indígena para el Desarrollo Autónomo (ECIDEA). Así mismo existe el Programa de Formación de Maestros indígenas especializados en educación intercultural y bilingüe en Iquitos (GASCHÉ, 2008); el Centro de Desarrollo Educacional Mapuche, fue creado en 1981 y constituye una propuesta que surge del análisis indígena en busca de una educación propia (Huechulaf, Ancalaf y Lienlaf: 2003); el modelo educativo Simiátug, es un modelo educativo autogestionario promovido por la Fundación Runacunapac YachanaHuasi (FRYH) que ha otorgado una aportación importante a la educación intercultural bilingüe en el ecuador, entre otros.
}

Dossiê Redes de Inter-aprendizaje: nuevas cartografías interculturales y algunas propuestas de transformación R. Articul.const.saber, 2020, v.5: e65682 
como occidentales (DIETZ, 2009) que han hecho grandes aportes a la educación intercultural bilingüe.

\section{El enfoque sintáctico de cultura y el Método Inductivo Intercultural: innovación pedagógica ante la diversidad cultural}

Frente a los serios obstáculos a los que se enfrenta la EIB, irrumpen metodologías y enfoques epistemológicos dialógicos y descolonizadores, que demuestran el potencial transformador que descansa en la co-teorización cultural ${ }^{7}$, la etnografía reflexiva, el diálogo de saberes y el Método Inductivo Intercultural, constituidos desde y con la participación de los mismo pueblos y comunidades indígenas (BERTELY, DIETZ Y DÍAZ, 2013; DE SOUSA, 2009, SARTORELLO, 2016, 2013).

El enfoque sintáctico de la cultura propuesto por Jorge Gasché concibe que la cultura se manifiesta en las actividades humanas. De este modo, el conocimiento indígena ${ }^{8}$ es un factor productivo funcional que participa en la actividad (GASCHÉ, 2008), que se actualiza constantemente en la cotidianeidad y que es expresada verbalmente en los discursos que acompañan a la actividad. Según GASCHÉ (2008), desde esta concepción de la cultura, la fuente del conocimiento indígena no son las personas tomadas aisladamente sino tomadas en su situación y su papel como actores, como productores.

De esta manera, el aula se acerca a los "hechos culturales" implícitos en las actividades tal y como suceden en la vida cotidiana de la comunidad, como hechos subjetivados y objetivados. De esta forma constituye un modelo sintáctico de producción de contenidos escolares e interculturales relacionado con la actividad productiva que transforma la naturaleza en cualquier sociedad y cultura.

\footnotetext{
${ }^{7}$ Se trata de una etnografía bajo un enfoque colaborativo en la cual se construyen aproximaciones teóricas y metodológicas desde las posturas culturalmente situadas en colaboradores indígenas (Stefano, 2013).

8 "Según el concepto del conocimiento indígena, la diferencia básica que actualmente lo distingue de manera desventajosa del conocimiento convencional, es que todavía no existen los materiales pedagógicos, ni en lo que se refiere a los currículos que lo clasificarían, graduarían y dosificarían, ni en lo que se refiere a los libros de clase, los libros de los maestros y las enciclopedias escolares que facilitarían el trabajo de los maestros y alumnos" (Gasché, 2008b: 207).
}

Dossiê Redes de Inter-aprendizaje: nuevas cartografías interculturales y algunas propuestas de transformación R. Articul.const.saber, 2020, v.5: e65682 
Este concepto de cultura sustenta al Método Inductivo Intercultural (MII) implementado por Jorge Gasché, Jesica Martínez y Carmen Gallegos en la Amazonía peruana y que en el caso de México, se ha extendido en los estados de Chiapas, Puebla, Michoacán y Oaxaca por medio de la Red de Educación Inductiva Intercultural (REDIIN) ${ }^{9}$. El Método permite apoyar la atención de la diversidad cultural a partir de una red que "pesca" conocimientos y valores indígenas a fin de crear un currículo específico para cada pueblo que, a su vez, articula estos conocimientos y valores con los escolares, garantizando la calidad, la pertinencia y la relevancia educativa.

Para lograr esto, el MII concibe que existen 4 fuentes del conocimiento indígena: niños, docentes, comunidad y obras antropológicas (Cfr. GASCHÉ, 2004) con los que se interaprende. También hace uso de un Calendario Socionatural donde se organizan y sistematizan las actividades comunitarias que se realizan durante el año y los conocimientos contenidos en ellas (Ver anexo 1). A la vez sirve como guía para orientar la actividad pedagógica bajo este enfoque. También se hace uso de la Matriz de Contenidos del Área Integradora Sociedad y Naturaleza que sirvió como una herramienta para la sistematización de la información otorgada por los niños y que podría apoyar en la planeación didáctica (Ver anexo 2). Ambas herramientas apoyan a sistematizar los conocimientos indígenas - que son dinámicos y cambiantes- que no se encuentran de la misma manera que los conocimientos escolares-universales, por lo que se utilizan con el fin de aprovechar pedagógicamente las actividades comunitarias (en este caso, las relacionadas con la pesca) así como su posible articulación y ampliación intercultural y generar, por medio del MII, contenidos escolares (comunitarios indígenas y escolares-universales) (UNEM, 2009: 85). Según GASCHÉ (2008):

(...) aun cuando el conocimiento indígena exige un esfuerzo previo a su uso pedagógico: el de objetivarlo notándolo por escrito, inventariándolo, sistematizándolo e integrándolo en manuales y materiales escolares decir convertirlo en un saber que existe en las mismas condiciones materiales que el saber escolar convencional y que, desde luego, puede ser manejadode la misma manera

\footnotetext{
${ }^{9}$ La Red de Educación Inductiva Intercultural (REDIIN) se encuentra conformada por Nodos Primarios: UNEM y Educadores Independientes de Chiapas, así como profesores-investigadores del CIESAS en convenio con el Instituto de Investigaciones de la Amazonía Peruana (IIAP). Nodos Secundarios: instituciones públicas que contribuyen al complimiento de los objetivo de mencionada Red.
}

Dossiê Redes de Inter-aprendizaje: nuevas cartografías interculturales y algunas propuestas de transformación R. Articul.const.saber, 2020, v.5: e65682 
en el aula de una existencia exclusivamente oral y arraigada en las mentes y los gestos, el conocimiento indígena, al parecer debe adquirir una existencia escrita para que sea conservado en libros y, de esa manera, alcance el mismo estatus material y operativo que el conocimiento escolar convencional (...) (GASCHÉ, 2008: 306-307).

El MII plantea que el conocimiento indígena explicitado y sistematizado debe cumplir dos funciones importantes: por un lado posibilidades de ser articularlo al conocimiento científico-escolar con eficiencia formativa $\mathrm{y}$, por el otro, traducirse en revaloración sociocultural. Como hemos visto, GASCHÉ (2008) expone una visión sintáctica de cultura de la cual se deriva la propuesta pedagógica que desarrolla sus contenidos a partir de una actividad. Esto supone que la cultura es entendida como las acciones que producen los seres humanos "en su proceso vivencial diario, en el cual crean sus medios de subsistencia, transformando la naturaleza, cooperando, interactuando y comunicándose entre ellos" (:314). En consecuencia, la cultura se define como el resultado de las actividades humanas, donde las fuentes de conocimiento son las personas tomadas en situación a partir de su papel como productores, es decir, como pescadores haciendo pesca o realizando alguna actividad derivada de la misma.

\section{Atención de la diversidad cultural: una propuesta pedagógica}

La realidad es que son escasos los materiales pedagógicos que faciliten el diálogo de saberes (DE SOUSA, 2006) entre el conocimiento escolar y los conocimientos indígenas en un mismo nivel de importancia. En este sentido, y para minimizar la desventaja mencionada y con base en el MII, este documento se plantea un primer esfuerzo por explicitar el conocimiento indígena relacionado con la pesca, con el fin de que el docente descubra de manera paulatina una alternativa que le permita partir del conocimiento indígena para apoyar los procesos pedagógicos y desarrollar competencias interculturales en los actores involucrados.

Para lograr lo anterior, el MII sugiere que el docente apoye a la explicitación de los conocimientos comunitarios, su profundización y organización curricular por medio de los cuatro ejes que estructuran el enfoque sintáctico de cultura. Los cuatro ejes son: objeto/recurso

Dossiê Redes de Inter-aprendizaje: nuevas cartografías interculturales y algunas propuestas de transformación

R. Articul.const.saber, 2020, v.5: e65682 
natural (¿qué?), medio ambiente/naturaleza (¿dónde?), transformación/técnica (¿con qué?, ¿cómo?), fin social/sociedad (¿para qué?). Estos ejes facilitan la articulación intercultural de los conocimientos comunitarios y escolares mediante un proceso que atiende lo genérico a través de lo específico, con el fin de explicitar los conocimientos implícitos en la acción comunitaria.

Con base en estos ejes, GASCHÉ (2008) propone una oración que expresa de manera concreta, pero genérica en qué consiste lo que se llama una actividad productora y actualizadora de cultura y conocimiento indígena: “el ser humano produce cosas yendo a la naturaleza a sacar sus materiales para transformarlos con el fin de satisfacer una necesidad (de forma social)" (:315).

Con base en la experiencia que se tuvo en la investigación, se sugiere que para lograr lo anterior, el primer paso que se propone es hacer uso de los datos otorgados por los niños, maestros y comuneros que fueron organizados en la Matriz de Contenidos (Ver anexo 2) y el Calendario Socionatural (Ver anexo 1). En función de la temporada del año en que se planeará la actividad, los docentes y los niños seleccionan una actividad para posteriormente seleccionar juntos una acción que defina la una unidad de aprendizaje. Por ejemplo: tomando en cuenta que se encuentran iniciando un nuevo ciclo escolar que generalmente se da en el mes de agosto, propondrán una actividad que corresponda a ese mes, para que los aprendizajes y conocimientos sean más significativos, contextualizados y acordes a la temporada. En este caso la oración quedaría de la siguiente manera: "vamos a la laguna a pescar camarón con el copo para comer y vender" que de acuerdo con el Calendario Socionatural construido en esta investigación, se ubica entre los meses de agosto-octubre (ver Anexo 1). Esta oración responde al medio ambiente/naturaleza (¿dónde?), Objeto/recurso natural (¿qué?), transformación/técnica (¿con qué?, ¿cómo?), fin social/sociedad (¿para qué?). Con esta oración se puede desarrollar actividades pedagógicas a partir de una actividad social. Con este método el aula se acerca a los "hechos culturales" implícitos en la actividad tal y como existen y se practican subjetivamente (ver GASCHÉ, 2008).

A continuación se presenta una propuesta de planeación que ejemplifica, en el caso de primer grado, la manera en que una actividad conformada con base en los cuatro ejes del modelo sintáctico de cultura, permite organizar y sincronizar los conocimientos implícitos de

Dossiê Redes de Inter-aprendizaje: nuevas cartografías interculturales y algunas propuestas de transformación R. Articul.const.saber, 2020, v.5: e65682 
las actividades comunitarias y los conocimientos escolares. Se eligió primer grado porque en esta investigación se identificó que los niños inscritos en los primeros años de escolarización $\left(1^{\mathrm{o}}\right.$ y $2^{\mathrm{o}}$ año) proporcionan datos más detallados de elementos culturales que tienen que ver con la pesca en comparación con los niños de grados superiores. Se espera que esta planeación sea un ejercicio que ayudé al docente a analizar la manera en que estos conocimientos pueden ser aprovechados para su articulación con los conocimientos escolares.

\title{
EJEMPLO DE PLANEACIÓN SEMANAL ${ }^{10}$
}

GRADO: $1^{\circ}$ BIMESTRE: I

NOMBRE DEL BLOQUE: Aprendiendo sobre el camarón

PROPÓSITOS DEL BLOQUE: Que el alumno conozca el hábitat, características, técnicas e instrumentos, así como los fines sociales de la pesca del camarón.

Vamos a la laguna a conocer cómo, dónde y con qué especies convive el camarón.

\section{EJES DEL MII: TERRITORIO (¿dónde?) "Vamos a la laguna..."}

\section{ACTIVIDAD PEDAGÓGICA ESPECÍFICA:}

\author{
1. En el salón de clase:
}

a) Dibujemos colectivamente en el pizarrón un mapa que indique cómo se llega a la laguna, ubicar y anotar los nombres y los lugares e indicadores de su localización durante el trayecto y sus características. Copiemos este mapa en los cuadernos; mapa que iremos mejorando y nos ayudará como guía durante el paseo.

b) Con el apoyo del docente, madres y padres de familia, elaboraremos en el pizarrón un crucigrama de especies lacustres que contenga los nombres del camarón y otros animales acuáticos que podemos encontrar en la laguna. Hagamos un crucigrama en ombeayiiüts y otro en español y mencionemos en cualquiera de estas dos lenguas lo que sabemos acerca de las características de cada una de ellas.

c) Borremos las palabras dejando solamente la primera letra de cada una de ellas como pista. ¿Qué letras quedaron? Copiemos el crucigrama para agregar especies y pistas después del paseo.

\section{APRENDIZAJES PROPIOS (en ombeayiiüt)}

-Territorio de la comunidad y los lugares sagrados destinados a la pesca.

\footnotetext{
${ }^{10}$ Esta propuesta de planeación es modificable y no se encuentra acabada, es decir, se encuentra sometida a las condiciones del contexto, por lo que cada una de las actividades expuestas pueden desagregar otras en los diversos ejes (territorio, recurso natural, técnica y fin social) como por ejemplo: la asignación de los territorios en la laguna para la pesca, la relación de la colocación del copo y la dirección del viento, la dirección del viento y el comportamiento de los animales, la importancia del cuidado de la laguna para evitar que haya plaga de medusas y agua mala que no permiten una pesca idónea del camarón y los cuidados que se debe tener para evitar accidentes, por mencionar algunos.
}

Dossiê Redes de Inter-aprendizaje: nuevas cartografías interculturales y algunas propuestas de transformación R. Articul.const.saber, 2020, v.5: e65682 
-Organización e Indicadores geográficos de la comunidad

-Caracterización del territorio de la comunidad

-Ubicación de los territorios para la pesca y lo que significan para la cosmovisión ikoot.

- Identificación en ombeayiiüts y español de las principales especies marinas que se encuentran en los territorios para la pesca.

-La importancia del cuidado de estas especies y la relación que guardan entre ellas.

- Importancia que se le asigna desde la cultura ikoot a cada una de esas especies.

\author{
APRENDIZAJES ESCOLARES (en ombeayiiüt con iniciación al castellano) \\ -Ubicación espacial. \\ -Indicadores geográficos (direcciones cardinales). \\ - Representación gráfica de datos. \\ -Utilización de referencias básicas (derecha, izquierda, lejos, cerca). \\ - Descripción de características. \\ -Ortografía. \\ -Escritura de palabras cortas en ambas lenguas. \\ -Resolución de crucigramas. \\ -Comparación. \\ -Cadena alimenticia
}

\title{
ACTIVIDAD PEDAGÓGICA ESPECÍFICA
}

2. Antes de salir de la escuela:

a) Recordemos que el día de mañana debemos orientarnos con base en los mapas, la posición del sol, la ubicación de

los puntos cardinales y otros indicadores en ombeayiiüts.

b) Pidamos al pescador y demás acompañantes que vayamos a la laguna y aprovechemos para interaprender.

\section{¡Escuchemos sus consejos!}

\section{APRENDIZAJES PROPIOS (en ombeayiiüt)}

-Formas propias de indicar direcciones y lugares en ombeayiiüt.

-La relación que guardan los puntos cardinales con la dirección del viento.

-Reconocer los conocimientos y consejos a las personas de la comunidad que se dedican a la actividad pesquera.

-Reconocer a la actividad pesquera como una de las más importantes de la cultura ikoot.

\section{APRENDIZAJES ESCOLARES (en ombeayiïit con iniciación al castellano)}

-Puntos cardinales (utilización de la rosa de los vientos).

\section{RECURSOS}

-Cuaderno.

-Lápiz.

-Borrador.

-Colores.

-Regla.

-Padre de familia/pescador.

-Padre y madre de familia voluntarios.

\section{ACTIVIDAD: Vamos a seleccionar camarón para preparar distintas comidas}

EJES DEL MII: RECURSO NATURAL (¿qué?) “...a pescar camarón...”

Dossiê Redes de Inter-aprendizaje: nuevas cartografías interculturales y algunas propuestas de transformación

$$
\text { R. Articul.const.saber, 2020, v.5: e65682 }
$$




\section{ACTIVIDAD PEDAGÓGICA ESPECÍFICA}

\section{Durante el paseo:}

a) Verifiquemos que atendimos los consejos del pescador, las madres y padres de familia y no nos falta nada para que nuestro paseo sea bueno y seguro.

b) Identifiquemos los puntos clave del territorio que orientan a la personas para llegar a la laguna.

c) Los señores y señoras nos platicarán acerca de sus trabajos en la pesca y otras actividades relacionadas. ¿En cuáles podemos participar niños y niñas?

Platiquemos cómo viven, cómo y de qué se alimentan, cómo y cuándo se reproducen cada una de las especies que conviven en la laguna y cuáles se comen, así como los tiempos del año en que no se debe pescar y por qué

\section{APRENDIZAJES PROPIOS (en ombeayiiüt)}

- Conocer más acerca de nuestro territorio

-Identificar físicamente los lugares sagrados dedicados a la pesca.

-Actividades que deben y no deben realizar los hombres y las mujeres en la laguna.

-Actividades de hombres y mujeres relacionadas con la pesca.

-Actividades de las niñas y los niños relacionadas com la pesca.

-Caracterización e identificación de las principales especies lacustres que se encuentran en los territorios para la

pesca

Tiempos de veda y la importancia de respetar las temporadas de reproducción de las especies.

-Identificar las consecuencias de la sobreexplotación pesquera.

-Caracterización e identificación en ombeayiiüts y español de las principales espécies lacustres que se encuentran en los territorios para la pesca.

-Tipos de camarones.

-Clasificación de los camarones

\section{APRENDIZAJES ESCOLARES (en ombeayiiiut con iniciación al castellano)}

-Indicadores territoriales de la localidad.

-Fauna local.

-El ciclo de la vida.

-Características de los animales.

-Cuidado del medio ambiente.

2. En la laguna:

a) Participemos en alguna actividad segura para las niñas y los niños relacionada con la pesca y selección del camarón en las orillas de la laguna.

b) Descansemos, dibujemos o anotemos algunas características de los animales que observamos en la laguna o lo que más nos gustó del paseo.

\section{En casa:}

a) Con lo aprendido en el paseo a la laguna y la ayuda de nuestra familia mejoremos nuestros crucigramas de especies lacustres en ombeayiiüts y español.

\section{*Término de jornada}

\section{APRENDIZAJES PROPIOS (en ombeayiiüt)}

Dossiê Redes de Inter-aprendizaje: nuevas cartografías interculturales y algunas propuestas de transformación R. Articul.const.saber, 2020, v.5: e65682 
-Identificación de las partes y funciones del cuerpo del camarón.

APRENDIZAJES ESCOLARES (en ombeayiïit con iniciación al castellano)

-Escritura de palabras en español y en ombeayiiüts.

-Arte.

-Imaginación.

-Habilidad verbal.

\section{En el salón de clases:}

a) Presentemos nuestros crucigramas y con la información recabada produzcamos sencillas adivinanzas.

b) Con el apoyo de las madres de familia formemos grupos en torno a las cubetas que contienen camarón y seleccionemos por tamaños los camarones.

c) Mientras realizamos la actividad hablemos de las funciones que cumple cada parte del cuerpo del camarón. El pescador, los acompañantes adultos y el docente complementaremos la información proporcionada.

d) Dibujemos un camarón y anotemos el nombre de las partes de su cuerpo y de los distintos platillos que pueden prepararse con estas partes.

\section{APRENDIZAJES PROPIOS (en ombeayiiüt)}

-Identificación de los diferentes platillos que se pueden hacer con las distintas partes del cuerpo del camarón, por ejemplo con la cabeza y la cola se hacen tortitas de camarón o mole de camarón, con el cuerpo ensaladas o comerlo solo como acompañante del queso, frijol y memelas.

-Características físicas que se debe de conocer para la selección del camarón con fines alimenticios.

-Reconocer la importancia del papel de la mujer ikoot en la preparación de los platillos.

\section{APRENDIZAJES ESCOLARES (en ombeayiiüt con iniciación al castellano)}

-Las adivinanzas.

- Escritura.

-Ortografía.

-Comparación.

-Los tamaños.

-La selección.

-Indicadores climáticos.

-Importancia de las partes del cuerpo.

-Identificación de las partes del cuerpo.

-Las partes y funciones del cuerpo de los animales

-Alimentación regional

-Importancia de comer sanamente

\section{En casa:}

a) Conversaremos con alguien que sepa pescar en la laguna y le preguntarán cómo se pesca, transporta, selecciona y conserva el camarón que se comen en la casa. Pediremos a su familiar que lleve un copo a la escuela.

\section{APRENDIZAJES PROPIOS (en ombeayiiü)}

-Conocer los cambios en la actividad pesquera en la historia de San Mateo del Mar.

-Identificación de los procedimientos, herramientas y técnicas que generalmente hace una persona para pescar camarón

-Reconocer quienes pueden usar y tocar las herramientas para la pesca

-Identificar los cuidados que debe tener la mujer con su cuerpo en relación a los espacios dedicados a la pesca.

Dossiê Redes de Inter-aprendizaje: nuevas cartografías interculturales y algunas propuestas de transformación

R. Articul.const.saber, 2020, v.5: e65682 
-Conocer los significados en relación a "ser hombre" o "ser mujer" que se circunscriben alrededor de las herramientas para la pesca. Por ejemplo, las mujeres no deben manipulas las herramientas para la pesca por que provocan que no haya buena pesca.

-Identificar la relación entre los indicadores climáticos (lluvia, calor y aire) con las temporadas de pesca. Por ejemplo: en temporada de lloviznas hay mucho camarón, pero cuando hay sequía escasea

\section{APRENDIZAJES ESCOLARES (en ombeayiüt con iniciación al castellano)}

-Estilos de vida

-Género

-Los roles

-Descripción de pasos

-Lenguaje

-Comunicación

\section{Recursos}

-Cuaderno.

-Lápiz.

-Borrador.

-Colores.

-Hojas blancas.

-Regla.

-Pizarrón.

-Gises.

-Padre de familia/pescador.

-Padres y madres de Familia/voluntarios.

-Canasto.

-Bicicleta.

-Cubetas con camarón.

*Término de jornada*

Descubramos cómo y con qué se pesca el camarón

Técnica (¿con qué?) “...con el copo...”

En el salón de clase:

a) Hablemos de lo que dibujamos y anotamos acerca de lo que saben nuestros familiares sobre la historia de la pesca, las mejores temporadas y el uso de las herramientas para pescar y conservar el camarón.

b) Con la compañía del pescador manipulemos el copo y descubramos cómo y con qué material se hace, cómo se usa y qué pueden hacer los niños y niñas para aprender a hacerlo o usarlo

Comentar:

-Qué es el copo y por qué es diferente a otras herramientas

-De qué material está hecho comparando la resistencia del material del copo y otros materiales no resistentes e inadecuados. -Quiénes y cómo se hace el copo

-Identificar las partes del copo

-Identificar las funciones de cada una de sus partes

-Cuál es el proceso de colocación y retiro

-Cómo se guarda

Dossiê Redes de Inter-aprendizaje: nuevas cartografías interculturales y algunas propuestas de transformación

R. Articul.const.saber, 2020, v.5: e65682 
c) Hagamos una actividad de nudo y trenzado con los materiales adecuados a nuestras manos.

\section{APRENDIZAJES PROPIOS (en ombeayiiüt)}

-Conocimiento de parte de la historia comunitaria

-Partes, funciones y utilización de las herramientas y estratégias de la actividad pesquera y formas de conservación del camarón.

- Herramientas para atrapar camarones

-Conocimiento general acerca de la pesca del camarón con el copo

-Conocimiento sobre:

* Materiales para la elaboración de la herramienta

* Peso aproximado de la herramienta

* Costos para la elaboración de la herramienta

*Técnicas de nudo y trenzado (cerrado/abierto).

*Otras técnicas de nudo y trenzado.

\section{APRENDIZAJES ESCOLARES (en ombeayiüit con iniciación al castellano)}

\section{-Habilidad oral}

-Memoria

-Reporte

-Identificar los cuerpos geométricos

-Conocer Unidades de medida (metro, centímetros, kilogramos).

-Hacer una secuencia.

-Habilidad motriz fina y gruesa.

-Memoria.

\section{En casa:}

a) Con la ayuda de nuestras madres y padres de familia participaremos en alguna actividad que tenga que ver con la pesca o preparación de un platillo como el caldo de camarón. Realizar un dibujo de la actividad realizada o la receta casera elaborada en casa para llevarlo a la escuela el día de mañana.

\section{APRENDIZAJES PROPIOS (en ombeayiiü)}

Interaprender la elaboración de nudos y trenzados con el apoyo de expertos y padres y madres de familia voluntarios.

\section{Preparemos un rico caldo de camarón para estar contentos y sanos}

Fin social (¿para qué?) “...para comer”

\section{En el salón de clase:}

a) Platiquemos de nuestros dibujos y recetas así como de cómo nos ayudaron las mujeres de la casa en la elaboración del platillo con camarón que disfrutamos en casa.

b) Pidamos a las madres de familia y al maestro que compartan y anoten en el pizarrón la receta del caldo de camarón, mientras la copiamos en los cuadernos.

c) Pasemos a voluntad al pizarrón y encerremos con un círculo las letras mayúsculas, subrayemos las minúsculas y tachemos las vocales.

d) ¡Fijémonos en los utensilios, ingredientes, cantidades y consejos para preparar el caldo y que alcance para todos!

Dossiê Redes de Inter-aprendizaje: nuevas cartografías interculturales y algunas propuestas de transformación 
e) Anotemos en el cuaderno el cálculo de la cantidad de los ingredientes de acuerdo al número de los comensales, nos basaremos en la lectura de los pasos de la receta anotada en sus cuadernos.

f) Verifiquemos que tenemos todo para hacer nuestro caldo de camarón.

\section{APRENDIZAJES PROPIOS (en ombeayiiü)}

-Procesos para la elaboración del caldo de camarón.

-Clasificación de las actividades de acuerdo al género.

-Trabajo femenino de la cultura ikoot.

-La autonomía femenina de la cultura ikoot.

-Conocimiento sobre las unidades de medida propias.

- Materiales necesarios para la preparación del caldo de camarón.

APRENDIZAJES ESCOLARES (en ombeayiüit con iniciación al castellano)

-Descripción de procesos.

-Función comunicativa y social de la lengua.

-Prácticas de lectura y escritura.

Identificación de mayúsculas, minúsculas y vocales.

-Colaboración.

-Respeto.

-Trabajo en equipo.

-Unidades de medida.

-Suma.

-Multiplicación.

-Cálculo.

\section{En una casa cercana a la escuela:}

a) Con la compañía de las madres de familia formaremos equipos para seleccionar los camarones.

b) Para la selección recordaremos lo que aprendimos al observar en la visita a la laguna.

c) Preparemos la cantidad de ingredientes suficientes para la elaboración del platillo y el número de comensales

d) Identificaremos grupalmente los utensilios y herramientas así como sus funciones para preparar los ingredientes de la receta.

e) Pongamos todo en el fogón o estufa de acuerdo a los consejos de las madres de familia y nuestra receta.

f) Veamos cómo se mueven las manecillas del reloj y cambia la posición del sol durante el tiempo de preparación y cocción del caldo de camarón.

g) Cuando esté listo el caldo comparemos el estado de los ingredientes y reconozcamos las diferencias entre camarón crudo y cocido.

\section{¿Disfrutaremos la comida preparada!}

*Término de la jornada*

\section{APRENDIZAJES PROPIOS (en ombeayiiuit)}

-Ingredientes que contiene del caldo de camarón.

-Utensilios y sus propiedades (cortar, mover, despegar, mezclar, calentar, enfriar, lavar, colar) al preparar la receta.

Dossiê Redes de Inter-aprendizaje: nuevas cartografías interculturales y algunas propuestas de transformación

R. Articul.const.saber, 2020, v.5: e65682 
-Identificación de platillos típicos regionales.

-Elaboración de recetas.

-Cantidades y propiedades de los ingredientes de la receta.

- Reciprocidad entre el hombre y la laguna.

-Concelebración.

-Colaboración y bien común.

-Buen vivir.

APRENDIZAJES ESCOLARES (en ombeayiiüt con iniciación al castellano)

-Selección.

-Identificación de tamaños.

-Comparación.

-Trabajo en equipo.

-Cálculo.

-Motricidad.

-Memoria.

-Mediciones y relaciones.

-Equivalencias.

-Gastronomía regional.

-Alimentación equilibrada.

-Propiedades alimenticias.

-La importancia de una buena alimentación.

-El reconocimiento de tener comida fresca y saludable.

-Importancia de la cocción de los alimentos.

3.En el salón de clases:

a) Formemos equipos y cada uno dibujará duplicadamente en tarjetas blancas los utensilios que se ocuparon para la realización del cado de camarón con el fin de elaborar un memorama y jugar con sus compañeros.

b) Investiguemos acerca de cómo cuidar la laguna para que las especies lacustres sean protegidas.

c) Realicemos carteles sobre lo que hemos aprendido y acerca de los cuidados de la laguna para pegarlos en los mercados, escuelas y calles de la comunidad.

d) Evaluemos nuestros trabajos e invitemos a los demás profesores, familias y comuneros a participar en el mejoramiento de nuestro Calendario Socionatural y la selección de nuestras próximas actividades.

e) Invitemos a los acompañantes de Chiapas para que nos ayuden a sembrar el MII en San Mateo del Mar, Oaxaca.

\section{APRENDIZAJES PROPIOS (en ombeayiiuit)}

-Integridad sociedad- naturaleza.

-Respeto a la madre tierra

-Analizar las formas en que las deidades se "enojan” y castigan cuando las cosas no se hacen bien.

-Reciprocidad entre la madre tierra y el hombre.

-Devolución de los interaprendizajes a la comunidad.

-Vitalizar la acción colectiva a favor de la integridad sociedad- naturaleza.

-Sedimentar el MII en la comunidad.

-Asegurar la colaboración y apoyo comunitario así como los interparendizaje entre todos.

\section{APRENDIZAJES ESCOLARES (en ombeayiïit con iniciación al castellan}

-Fortalecer la REDIIN.

-Cuidado del medio ambiente.

Dossiê Redes de Inter-aprendizaje: nuevas cartografías interculturales y algunas propuestas de transformación

R. Articul.const.saber, 2020, v.5: e65682 
-El memorama.

-El cartel.

-La escucha.

-La pregunta.

-Colaboración, cooperación y trabajo en equipo.

Tomando en cuenta la anterior propuesta, la concepción de currículo de primaria del MII se desarrolla pensando que las actividades pedagógicas se dan a partir de una actividad social, la cual se fue desagregando en otras actividades y de manera transversal llevan al tratamiento de conocimientos propios y escolares. Las actividades sugeridas en la planeación proporcionan a los docentes una idea de la manera en que la escuela puede abrir sus puertas a la comunidad.

Se debe considerar que la planeación anteriormente expuesta, elaborada por mí por la imposibilidad de elaborarla con los docentes en tiempos de movilización magisterial, no es estática. Todo lo contrario, el docente puede identificar diversos conocimientos en términos interculturales para producir una planeación dinámica y proponer situaciones didácticas áulicas y extra áulicas in situ que ayuden a la articulación de los conocimientos escolares y los comunitarios.

Además esta planeación debe considerar la necesidad de secuenciar los aprendizajes en función del grado escolar y las etapas del desarrollo cultural de las niñas y los niños, además de involucrarlos en la actividad por conducto de la acción. En términos pedagógicos, el docente debe planear y gestionar los procesos para que los cuales los niños puedan observar y hacer la actividad en cuestión. Esto traerá consigo conocimientos significativos, el interés de los niños y niñas por investigar al observar o hacer la actividad, el reconocimiento del conocimiento comunitario y el fortalecimiento de la identidad ikoot.

El uso de la lengua indígena en este ejercicio de planeación juega un papel fundamental. Ésta articula lengua y cultura, considera las funciones comunicativas y usos sociales del lenguaje y encierra una visión del mundo (GASCHÉ, 2008). El ombeayiiüts no solamente es una serie de códigos y signos que permiten la comunicación, sino una lengua que contiene los significados, epistemologías, valores y conocimientos culturales sustantivos que ayudan a entender la cosmovisión del pueblo ikoot. En este caso el ombeayiiüts es la

Dossiê Redes de Inter-aprendizaje: nuevas cartografías interculturales y algunas propuestas de transformación R. Articul.const.saber, 2020, v.5: e65682 
lengua materna de la mayoría de los niños del primer grado, lo cual les da la oportunidad de incursionar desde su mundo ikoot en los significados, epistemologías, valores y conocimientos pesqueros. Pero además, el aprendizaje del castellano como segunda lengua se apoya en el horizonte cultural desde la racionalidad ikoot. Según GASCHÉ (2008):

... en la medida en que el castellano constituye de todas maneras un objetivo de aprendizaje escolar, usar categorías sintácticas que también son las del castellano, puede aparecer además como un medio adecuado para traducir la realidad indígena y las reivindicaciones que de ella derivan a una forma de explicitación y explicación que es inmediatamente asequible a los miembros de la sociedad dominante, y que, desde luego, expresa el universo sociocultural indígena que queda generalmente replegado y encerrado detrás de las barreras de su lengua y fundamentalmente incomprendido por los observadores exteriores ignorantes de la lengua indígena... (2008: 319)

El manejo del ombeayiiüts como lengua materna y la adquisición del castellano como segunda lengua -en la mayoría de los casos- podrían ayudar relacionar los conocimientos y valores interculturales que se encuentran en el universo del niño ikoot. Por su parte el docente deberá ir creando estrategias emergentes que apoyen a la explicitación de los conocimientos comunitarios.

\section{Conclusiones}

Con el ejercicio de la planeación puede comprobarse la importante papel de la alfabetización territorial (BERTELY, 2007b) en contextos socioculturalmente diversos; esto por conducto de la actividad o en su defecto, a través del aprendizaje observacional (ROGOFF, 2012). Por lo tanto, esta planeación puede funcionar tanto con los niños y niñas que realizan algunas actividades relacionadas con la actividad pesquera, como con los niños y niñas que no la realizan, pero que gracias al aprendizaje observacional tiene conocimientos sobre dicha actividad, adquiridos en la vida cotidiana por medio de la experiencia.

En esta tónica se pensó que esta planeación podría ayudar al docente para:

1. Articular los conocimientos escolares con los conocimientos comunitarios.

2. Profundizar en los conocimientos escolares a partir de los conocimientos comunitarios.

Dossiê Redes de Inter-aprendizaje: nuevas cartografías interculturales y algunas propuestas de transformación R. Articul.const.saber, 2020, v.5: e65682 
3. Incitar la iniciación hacia la investigación social en los niños y niñas

4. Reconocer la relevancia e importancia de la práctica de los conocimientos comunitarios

5. Hacer conciencia de la importancia de la carga cultural que tiene la lengua ombeayiiüts para entender la cosmovisión de su pueblo.

6. Desarrollar habilidades didácticas que permitan la sincronización de los actores educativos (niños, padres de familia, docentes y directivos).

7. Fortalecer la identidad ikoot

Para poder lograr estos objetivos, los docentes deberán contar con competencias interculturales a través de la gestión de la diversidad basada en una concepción sintáctica de la cultura. Lo anterior derivará un proceso pedagógico apuntalado en las actividades sociales comunitarias que colocará en el centro del proceso pedagógico la cultura de los ikoots. Para desfortuna de las niñas y los niños, el proceso de escolarización formal ha disminuido la oportunidad de que este tipo de aprendizaje sea más cotidiano e intenso para ellas y ellos, ya que el hecho de asistir a la escuela reduce el tiempo que destinan a la participación y colaboración en las actividades comunitarias y, por lo tanto, tienen menos oportunidades de aprender a hacer por medio de la observación y la actividad práctica en colaboración con pares más capaces.

Es importante reconocer que esta propuesta apuntala la idea de sacar a la escuela hacia la comunidad, hacia los lugares donde se encuentran las actividades comunitarias, que los niños observen, participen y escriban sobre ellas. La escuela debe ser el puente para que los niños y niñas desarrollen conocimientos, habilidades y actitudes que les serán necesarios para vivir en la comunidad y que incluya lo que hasta ahora ha excluido: el aprendizaje práctico y discursivo de los saberes de la comunidad (GASCHÉ, 2008), con ello se concretiza el carácter intercultural de la escuela.

De esta manera se vuelve posible lograr los objetivos curriculares nacionales y a la vez transverzalizarlos con los conocimientos indígenas en su forma viva y practicada en la comunidad. Lo anterior transforma la relación que se tiene con el conocimiento, ya que el docente y el estudiante se identifican con el comunero que realiza la actividad, pero además con los conocimientos circunscritos en ella, por lo que el conocimiento se vuelve significativo.

Dossiê Redes de Inter-aprendizaje: nuevas cartografías interculturales y algunas propuestas de transformación R. Articul.const.saber, 2020, v.5: e65682 
Este ejercicio además de atender la pertinencia y relevancia educativa es el punto de partida hacia una pedagogía intercultural y políticamente pertinente (GASCHÉ, 2008).

Dossiê Redes de Inter-aprendizaje: nuevas cartografías interculturales y algunas propuestas de transformación R. Articul.const.saber, 2020, v.5: e65682 


\section{Bibliografía}

BERTELY, M. y GASCHÉ, J. (Coord.). Interaprendizajes entre indígenas. De cómo las y los educadores pescan conocimientos y significados comunitarios en contextos interculturales. México: CIESAS-REDIIN y UPN. (2011).

BERTELY, M. DIETZ, G. \& DÍAZ, M. Debates sobre interculturalidad y multiculturalismo en educación, Estado del Conocimiento Área 12. México: COMIE. (2013).

BERTELY, M. (Coord.). Modelo Curricular de Educación Intercultural Bilingüe UNEM. México: UNEM / ECIDEA / CIESAS / IIAP / OEI / Ediciones alcatraz. (2009).

Conflicto intercultural, educación y democracia activa en México. Cuidadanía y derechos indígenas en el movimiento pedagógico intercultural bilingüe en Los Altos, la Región Norte y la Selva Lacandona de Chiapas. CIESAS/Fondo editorial de la Universidad Católica del Perú: México. (2007).

DE SOUSA, B. Una epistemología del sur. La reinvención del conocimiento y la emancipación social. México: CLACSO. (2009).

DIETZ, G. Y MATEOS, L. Interculturalidad y educación intercultural en México. Un análisis de los discursos nacionales e internacionales en su impacto en modelos educativos mexicanos. México: SEP/CGEIB. (2019).

DIETZ, G. Del multiculturalismo a la interculturalidad y diversidad en educación. Una aproximación antropológica. México: FCE. (2010).

. Los actores indígenas ante la -interculturalización de la educación superior en

México. Revista Latinoamericana de Educación Inclusiva. (2009). En: http://www.rinace.net/rlei/numeros/vol3-num2/art4.html

GASCHÉ, J. Niños, maestros, comuneros y escritos antropológicos como fuentes de contenidos indígenas escolares y la actividad como punto de partida de los procesos pedagógicos interculturales: un modelo sintáctico de cultura. En: Gasché, J., Bertely, M. y Podesta, R. (Eds.). Educando en la diversidad. Investigaciones y experiencias educativas interculturales y bilinguies. México: Paidos. (2004).

Las motivaciones políticas de la educación intercultural indígena. ¿Hasta dónde abarca la interculturalidad? En GASCHÉ, J., BERTELY, M. Y PODESTÁ, R. (Eds.), Educando en la diversidad. Investigaciones y experiencias educativas interculturales $\mathbf{y}$ bilingües (pp. 367-397). Quito: AbyaYala. (2008).

HETCH, A. Y LONCON, E. (Comp.). Educación Intercultural Bilingüe en América Latina y el Caribe: balances, desafíos y perspectivas. Chile: Fundación Equitas. (2011).

Dossiê Redes de Inter-aprendizaje: nuevas cartografías interculturales y algunas propuestas de transformación R. Articul.const.saber, 2020, v.5: e65682 
JIMÉNEZ, Y. Cultura comunitaria y escuela intercultural. Más allá de un contenido escolar. México, DF:SEP-CGIB. (2009).

MALDONADO, B. Y MALDONADO, C. Educación e interculturalidad en Oaxaca: avances y desafíos. Sinéctica no.50 Tlaquepaque ene./jun. 2018. (2018). Consultado en: http://www.scielo.org.mx/scielo.php?script=sci_arttext\&pid=S1665-109X2018000100006

ROGOFF, B. Learning eithout lessons: opportunities tu expland knowledge. Infancia y aprendizaje. (2012) En:

http://www.tandfonline.com/doi/pdf/10.1174/021037012800217970

SARTORELLO, S. Política, epistemología y pedagogía: el Método Inductivo Intercultural en una escuela tseltal de Chiapas, México. En Revista LiminaR Estudios Sociales y Humanísticos, XIV, núm. 1, enero-junio de 2016, México, p. 121-143. (2016). Consultado en: http://www.scielo.org.mx/scielo.php?script=sci_arttext\&pid=S1665-

$\underline{80272016000100009}$

Conflicto, colaboración y co-teorización en un proceso intercultural de diseño curricular en Chiapas. Tesis doctoral sin publicar. Universidad Iberoamericana. México. (2013).

. Una perspectiva crítica sobre interculturalidad y educación intercultural bilingüe: El caso de la Unión de Maestros de la nueva educación para México (UNEM) y educadores independientes en Chiapas. Revista Latinoamericana para la Educación Inclusiva. (2009). En: http://www.rinace.net/rlei/numeros/vol3-num2/art5.pdf

SCHMELKES, S. La interculturalidad en la educación básica. Ponencia presentada en el contexto de la Segunda Reunión del Comité Intergubernamental del Proyecto Regional de Educación para América Latina y el Caribe (PRELAC). (2006). Consultado en: http://cresur.edu.mx/cresur/app_guias/app_int/articulos_academicos/interculturalidad_educa cion_basica.pdf

Dossiê Redes de Inter-aprendizaje: nuevas cartografías interculturales y algunas propuestas de transformación R. Articul.const.saber, 2020, v.5: e65682 


\section{Anexos}

Anexo 1. Calendario Socionatural sobre actividad pesquera de la cultura ikoot de San Mateo del Mar.

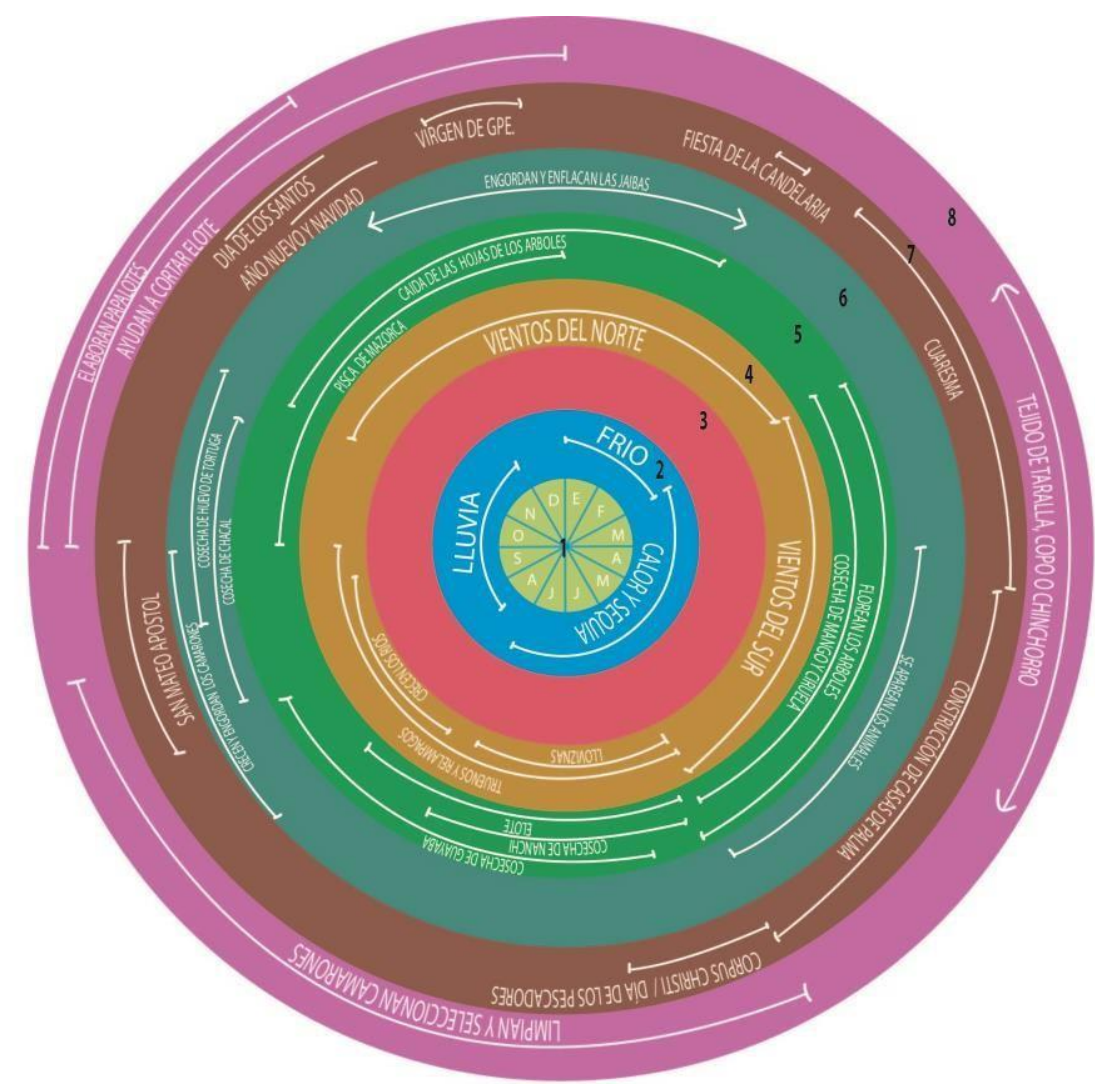

Elaboración

Fuente: propia

1. MESES OCCIDENTALES

2.TEMPORADAS

3. MESESINDIGENAS

4. INDICADORES CLIMATICOS

5. INDICADORESVEGETALES

6.INDICADORES ANMALES

7.ACTIVIDADES DELOS COMUNEROSY COMUNERAS

8. ACTIVDADES DELOS NINNOSYNINAS

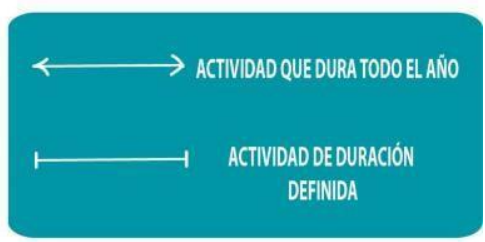

Dossiê Redes de Inter-aprendizaje: nuevas cartografías interculturales y algunas propuestas de transformación R. Articul.const.saber, 2020, v.5: e65682 
Anexo 2. Matriz de contenidos del Área Integradora Sociedad y Naturaleza relacionados con la pesca realizada con base en datos proporcionados por los niños de la Escuela Primaria Bilingüe Adolfo López Mateos.

\begin{tabular}{|c|c|c|c|}
\hline Territorio-naturaleza & $\begin{array}{l}\text { Recurso natural- } \\
\text { producto }\end{array}$ & Trabajo-técnica & Fin social-sociedad \\
\hline \multirow{3}{*}{$\begin{array}{l}\text { Mar muerto } \\
\text {-Hay palos para amarrar la } \\
\text { tarraya y el copo } \\
\text {-No hay olas }\end{array}$} & Tixem (camarón) & Copo & $\begin{array}{l}\text {-Venta en el mercado } \\
\text {-Consumo familiar } \\
\text { (caldo de camarón) }\end{array}$ \\
\hline & Jaiba & Tarraya & $\begin{array}{l}\text {-Venta en el mercado } \\
\text {-Consumo familiar } \\
\text { (hervido con sal) }\end{array}$ \\
\hline & Pulpo & Tarraya & $\begin{array}{l}\text {-Consumo familiar } \\
\text { (ensalada) }\end{array}$ \\
\hline $\begin{array}{l}\text { Laguna } \\
\text {-No hay olas }\end{array}$ & Camarón & Tarraya & $\begin{array}{l}\text {-Venta } \\
\text {-Consumo familiar }\end{array}$ \\
\hline \multirow{4}{*}{$\begin{array}{l}\text { Mar vivo } \\
\text {-No hay palos } \\
\text {-Olas grandes }\end{array}$} & Pulpo & \multirow[t]{3}{*}{ Tarraya } & -Consumo familiar \\
\hline & Pescado & & $\begin{array}{l}\text {-Venta } \\
\text {-Consumo familiar } \\
\text { (pescado frito y caldo } \\
\text { de camarón) }\end{array}$ \\
\hline & Medusas & & \\
\hline & Huevo de tortuga & $\begin{array}{l}\text { Recolección en la } \\
\text { playa }\end{array}$ & -Venta \\
\hline
\end{tabular}

Submetido em 17 de setembro de 2020.

Aceito em 13 de novembro de 2020.

Publicado em 01 de dezembro de 2020.

Dossiê Redes de Inter-aprendizaje: nuevas cartografías interculturales y algunas propuestas de transformación R. Articul.const.saber, 2020, v.5: e65682 